\title{
Development of swine-specific DNA markers for biosensor-based halal authentication
}

\author{
M.E. Ali ${ }^{1,3}$, U. Hashim ${ }^{1}$, M. Kashif ${ }^{1}$, S. Mustafa ${ }^{2}$, Y.B. Che Man ${ }^{2}$ and \\ S.B. Abd Hamid ${ }^{3}$ \\ ${ }^{1}$ Institute of Nano Electronic Engineering, Universiti Malaysia, \\ Kangar, Perlis, Malaysia \\ ${ }^{2}$ Institute of Halal Products Research, Universiti Putra Malaysia, \\ Serdang, Selangor, Malaysia \\ ${ }^{3}$ Nanotechnology and Catalysis Research Center, Universiti Malaysia, \\ Kuala Lumpur, Malaysia \\ Corresponding author: M.E. Ali \\ E-mail: eaqubali@gmail.com
}

Genet. Mol. Res. 11 (2): 1762-1772 (2012)

Received October 6, 2011

Accepted March 12, 2012

Published June 29, 2012

DOI http://dx.doi.org/10.4238/2012.June.29.9

\begin{abstract}
The pig (Sus scrofa) mitochondrial genome was targeted to design short (15-30 nucleotides) DNA markers that would be suitable for biosensor-based hybridization detection of target DNA. Short DNA markers are reported to survive harsh conditions in which longer ones are degraded into smaller fragments. The whole swine mitochondrialgenome was in silico digested with AluI restriction enzyme. Among $66 \mathrm{AluI}$ fragments, five were selected as potential markers because of their convenient lengths, high degree of interspecies polymorphism and intraspecies conservatism. These were confirmed by NCBI blast analysis and ClustalW alignment analysis with 11 different meatproviding animal and fish species. Finally, we integrated a tetramethyl rhodamine-labeled 18-nucleotide AluI fragment into a 3-nm diameter citrate-tannate coated gold nanoparticle to develop a swine-specific hybrid nanobioprobe for the determination of pork adulteration in $2.5-\mathrm{h}$ autoclaved pork-beef binary mixtures. This hybrid probe detected as low as $1 \%$ pork in deliberately contaminated autoclaved pork-beef binary
\end{abstract}


mixtures and no cross-species detection was recorded, demonstrating the feasibility of this type of probe for biosensor-based detection of pork adulteration of halal and kosher foods.

Key words: In silico digestion; Intra- and interspecies polymorphisms; Restriction enzyme; Mitochondrial genome; Amplicon length; Protein coding genes

\section{INTRODUCTION}

DNA molecules that are exposed to water and/or heat will begin to breakdown into smaller and smaller fragments due to bacterial, biochemical, and oxidative processes operating under natural environmental conditions (Butler, 2005, 2006). A number of studies have demonstrated that successful analysis of degraded DNA specimens or compromised forensic evidence is improved with the use of smaller-sized PCR amplicons, typically $\leq 150$ bp in length (Butler, 2006; Bielikova et al., 2010). Usually, the smaller the amplicon length, the better the recovery will be (Aboud et al., 2010; Ali et al., 2011a,b). High sensitivity in the PCR-RFLP assay for the detection of pork adulteration in commercial products is reported to be achieved with the reduction of amplicon length (Bielikova et al., 2010; Ali et al., 2011b). However, the PCR procedure has its limitations in reducing the amplicon length. Too much reduction of amplicon length often compromises specificity, producing artifacts in the final results (Hird et al., 2006; Ali et al., 2011b). On the other hand, shorter-sized DNA probes (1530 nucleotides) are suitable for sensor-based hybridization detection, a promising tool but still in inception (Kim et al., 2011; Ali et al., 2011c,d, 2012).

Multicopy nuclear DNAs, such as the variable number of tandem repeat (VNTR) and short tandem repeat (STR), have long been the targets for identifying individuals in forensic investigations (Mozayani and Noziglia, 2010). VNTRs are the locations of tandemly repeated sequences in the genome and often show variation in length and number of repeats between individuals. VNTR sequences can be amplified using primers targeting the flanking regions or digested by restriction enzymes. After recovery by PCR or RFLP analysis, a unique pattern of VNTR bands can be obtained, which provides useful information for identifying an individual. Except in identical twins, the VNTR profiles of two different individuals are unlikely to be the same. However, targeting VNTR often produces very long amplicons, which cannot be recovered from severely degraded specimens (Mozayani and Noziglia, 2010).

STRs are 2-5-nucleotide tandem repeats of nuclear DNA and are better substitute of relatively bulky VNTR sequences (Mozayani and Noziglia, 2010). STRs are highly polymorphic and capable of generating typing results from very little material through multiplex amplification using PCR (Butler, 2005). Commercial STR kits can generate amplicons in the range of 100-450 bp (Butler, 2006). The development of miniSTR-PCR assays, that effectively cut the amplicon lengths within $150 \mathrm{bp}$, significantly increases target recovery from highly degraded samples (Butler, 2006; Hill et al., 2006; Luo et al., 2011). However, miniSTR-PCR assays also need a considerably longer amplicons $(\geq 100 \mathrm{bp})$, which may not survive in a harsh environment, calling for the need for more stable markers to handle extremely degraded samples.

An alternative source of candidate markers is the mitochondrial (mt) genome. Mitochondria are very small organelles found outside the cell nucleus and within the cytoplasm. 
Mt-DNAs are circular and hence more stable than linear nuclear DNAs. They are present in multiple copies in each mitochondrion and several copies of mitochondria are present per cell (Mozayani and Noziglia, 2010; Luo et al., 2011). Mt-genes rarely undergo recombination (Luo et al., 2011). Thus, targeting mt-genes should ensure available targets even in extremely degraded forensic samples. However, mt-genomes are maternally inherited and consequently provide information only in relation to maternal background (Mozayani and Noziglia, 2010).

Although mt-DNAs cannot discriminate individuals like nuclear-STR and VNTR sequences can, mt-genes contain huge interspecies polymorphism and have proven to be effective in distinguishing species with a great efficiency (Ali et al., 2011a,b; Doosti et al., 2011; Yusop et al., 2011). The mt-genomes of all bilateralian animals contain 13 protein-coding genes, which encode proteins involved in oxidative phosphorylation machinery: cytochrome oxidase subunits 1, 2, and 3 (CO1-CO3); cytochrome $b$ subunit (cytB), NADH dehydrogenase subunits 1, 2, 3, 4, 4L, 5, and 6 (ND1-ND6, ND4L), and ATPase subunits 6 and 8 (ATP6 and ATP8). The mt-genome also contains 2 ribosomal RNA genes (12S and 16S rRNAs) and 22 transfer RNA (tRNA) genes (Luo et al., 2011). The inter- to intra-species gap (barcode gap) of all the genes of mt origin is greater than 10, which makes them effective markers for species differentiation in foods and feeds. Species-specific PCR assays targeting shorter-sized amplicons of mt-genes are reported to be more effective than the longer ones (Che Man et al., 2010; Ali et al., 2011a,b; Yusop et al., 2011).

In this study, we targeted the swine mt-genome to design markers for biosensor-based identification of pork adulteration in halal and kosher meats and meat products. Biosensorbased identification of DNA targets needs ssDNA probes whose lengths (15-30 bp) are almost equivalent to those of PCR primers (Kim et al., 2011; Ali et al., 2011c,d, 2012). DNA markers of these lengths should ensure higher stability than the comparatively longer PCR amplicons in harshly processed meat and meat products. Five DNA markers of mt origin were theoretically developed and one of them was tested for biosensor applications by designing a hybrid nanobioprobe, which was composed of a 3-nm gold nanoparticles and 18-nt fluorophore-labeled oligo-probe of swine-mitochondrial origin.

\section{MATERIAL AND METHODS}

\section{Probe design}

Sus scrofa (pig) mt-genome (16, 613 bp, GenBank accession No. AF034253.1) was retrieved from NCBI (National Center for Biotechnology Information; www.ncbi.nlm.nih.gov) and was analyzed for $A l u \mathrm{I}$ sites by NEB cutter version 2 (www.tools.neb.com/NEBcutter2) and REBASE (www.neb.com/rebase/rebase.html) softwares. A total of 66 sites were found (data not shown). Five of these fragments, as shown in Table 1, were preliminary selected as potential candidates of swine-specific markers because of their convenient lengths for biosensor applications (Kim et al., 2011). Analysis by NCBI basic local alignment search tool (BLAST) for highly similar sequences against non-redundant nucleotide collections revealed that these fragments were specific for the pig mt-genome. We retrieved the mt-genomes of common meat providing animals [Bos taurus (cow), Gallus gallus (chicken), Ovis aries (sheep), Capra hircus (goat), Cervus nippon (deer)] and fish species [Gadus morhua (shrimp), Thunus orientalis (tuna), Sepia officinalis (cuttlefish), Crenicichla minuano (cichlid), and Alosa 
sapidissima (shad)] from NCBI data base and aligned them side by side with the selected AluI-cut fragments using publicly available ClustalW alignment tool (www.genome.jp/tools/ clustalw/). All fragments demonstrated a high degree of specificity for the pig mt-genome and dissimilarities with the others.

\begin{tabular}{lcl}
\multicolumn{2}{l}{ Table 1. List of $A l u$ I fragments selected for biosensor applications. } \\
\hline Fragment size (nucleotides) & Position in mt-genome & Sequences (5'-3') \\
\hline 24 & $2504-2527$ & CTA GAT ACC CCG AAA CCA GAC GAG \\
25 & $975-9774$ & CTT ATG TGT TTA CAC TGC TAG TAA G \\
17 & $12282-12297$ & CTT CAT AGG GGC CAC AG \\
18 & $13589-13606$ & CTG CAG CAG GAAAAT CAG \\
27 & $15768-15794$ & CTA CGG TCA TCA CAA ATC TAC TAT CAG \\
\hline
\end{tabular}

\section{Development of swine-specific hybrid nanobioprobe}

The swine-specific hybrid nanobioprobe was developed according to the literature (Ali et al., 2011d,e). Briefly, custom-made oligo probes, as shown in Table 2, were incubated overnight at $20^{\circ} \mathrm{C}$ in a shaking water bath in a ratio of 3 oligos per particle to ensure that each particle was conjugated to at least one oligo molecule. To improve the stability and consequent hybridization efficiency, the oligo particle conjugates were aged in $0.1 \mathrm{M} \mathrm{NaCl}, 10 \mathrm{mM}$ PBS, pH 7.4, for $16 \mathrm{~h}$ according to the literature (Mirkin et al., 1996; Ali et al., 2011d). The nanoparticle probes were separated from unbound oligos by three times centrifugation at 50,000 rpm for $25 \mathrm{~min}$. The final product was a loose red pellet, which was reconstituted in $10 \mathrm{mM}$ PBS. The number of oligo molecules per particle was determined by 2-mercaptoethanol digestion according to a previously established procedure (Maxwell et al., 2002). The 3-nm diameter citrate-tannate coated gold nanoparticles were prepared according to Ali et al. (2011d).

\begin{tabular}{ll} 
Table 2. Probe and target oligo sequences used. \\
\hline \begin{tabular}{ll}
\hline Name & Sequences $\left(5^{\prime}-3^{\prime}\right)$ \\
\hline Porcine-probe & TMR-A - CTG CAG CAG GAA AAT CAG-A6-(CH $\left.{ }_{2}\right)_{6}$ SH $^{-}$ \\
Porcine-target (complementary) & CTG ATT TTC CTG CTG CAG \\
Porcine-target (single-mismatched) & CTG ATT TTC TTG CTG CAG \\
Porcine-target (non-complementary) & TTA CCC GGT CCA TCT ACT \\
\hline
\end{tabular}
\end{tabular}

\section{Specificity test of the nanobioprobe developed}

An aliquot of the purified nanoparticle probes was diluted to $10 \mathrm{nM}$ with hybridization buffer ( $90 \mathrm{mM} \mathrm{KCl}, 10 \mathrm{mM}$ Tris, $\mathrm{pH}$ 8.0). To determine specificity, the probes were incubated with a 4-fold excess $(60 \mathrm{nM})$ of complementary, non-complementary, and single-mismatch targets (Table 2) at $70^{\circ} \mathrm{C}$ for 5 min to allow strand separation and then at $40^{\circ} \mathrm{C}$ for $30-60 \mathrm{~min}$ to allow hybridization.

\section{Specificity and sensitivity tests in mixed meats}

Separate aliquots of $100 \mathrm{~g}$ pork-beef binary admixtures were prepared in a ratio of 100:0, 50:50, 25:75, 10:90, 5:95, 3:97, 1:99, and 0:100 fresh pork and beef. The mixtures were 
autoclaved at $120^{\circ} \mathrm{C}$ for $2.5 \mathrm{~h}$ and DNAs were extracted from $100 \mathrm{mg}$ samples in triplicate using the MasterPure ${ }^{\mathrm{TM}}$ DNA Purification kit (Epicenter Biotechnologies, Madison, USA) following the protocol optimized and supplied by the manufacturer. The purity and concentration of the extracted DNA samples were checked with an Eppendorf UV-vis Biophotometer (Eppendorf, Hamburg, Germany) and the following formulas (Ali et al., 2011c) were used to calculate purity and concentration:

DNA Purity $\left(\mathrm{A}_{260} / \mathrm{A}_{280}\right)$ :

$\left(\mathrm{A}_{260}\right.$ Reading-A $\mathrm{A}_{320}$ Reading $) /\left(\mathrm{A}_{280}\right.$ Reading- $\mathrm{A}_{320}$ Reading $)$

DNA concentration $(\mu \mathrm{g} / \mathrm{mL})$ :

$\left(\mathrm{A}_{260}\right.$ Reading- $\mathrm{A}_{320}$ Reading) x Dilution Factor x 50

The purity $\left(\mathrm{A}_{260} / \mathrm{A}_{280}\right)$ of all DNA samples used in subsequent experiments was 1.95-2.0. The extracted total DNA $(500 \mu \mathrm{g} / \mathrm{mL})$ was digested with $A l u \mathrm{I}$ (New England Biolabs, UK) restriction enzyme. The digestions were performed in a total volume of $1 \mathrm{~mL}$ containing $600 \mu \mathrm{L}$ total DNA, $300 \mathrm{U}$ restriction enzymes and $100 \mu \mathrm{L}$ digestion buffer (New England Biolabs) for 1 $\mathrm{h}$ at $37^{\circ} \mathrm{C}$ in a shaking water bath. The digestion was stopped by heating the mixture at $65^{\circ} \mathrm{C}$ for $10 \mathrm{~min}$ (Ali et al., 2011b). The final volume was adjusted with $18.2 \Omega$ Millipore water.

The hybridization reaction was performed in a total volume of $2.5 \mathrm{~mL}$ in triplicate with $10 \mathrm{nM}$ probes and $60 \mu \mathrm{g} / \mathrm{mL}$ AluI-digested mixed DNA according to Ali et al. (2011d). For the study of hybridization kinetics and the generation of standard curve, $10 \mathrm{nM}$ probes and various concentrations (Table 3) of AluI-digested raw-pork DNA were incubated in a 2.5 -mL reaction volume at $40^{\circ} \mathrm{C}$ for $60 \mathrm{~min}$. The concentration was adjusted with the hybridization buffer.

\section{Fluorescence measurement}

The emission spectra were collected in a $10-\mathrm{mm}$ cuvette with a $2-\mathrm{mL}$ volume in a PerkinElmer LS55 fluorescence spectrometer with excitation at $545 \mathrm{~nm}$. Each spectrum had an average of 5 scans at the scan speed of $200 \mathrm{~nm} / \mathrm{min}$ with a $5-\mathrm{nm}$ slit width. The background was subtracted by replacing the sample with $2: 1$ ratio of $10 \mathrm{mM}$ PBS and hybridization buffer. For the determination of limit of detection, a series of fluorescence spectra were obtained in triplicate and average fluorescence intensity at $579 \mathrm{~nm}$ was plotted as a function of target concentration.

\section{RESULTS AND DISCUSSION}

\section{Probe design}

The specificity of the mt-DNA markers depends on its intraspecies similarities and interspecies dissimilarities (Che Man, et al., 2010). NCBI BLAST analysis as well as ClustalW alignment analysis showed that the designed markers carried a high degree of interspecies polymorphism and intraspecies conservatism. The higher the ratio of inter- to intraspecies dissimilarities, the better the probe will be for discriminating closely related species (Luo et al., 2011). All the markers developed contained at least $10 \%$ mismatches with closely related species as revealed by alignment analysis. It has been shown that the effects of single mismatch on the 
primer binding region of the PCR template have little influence on the efficiency of PCR but the presence of two or more mismatches drastically reduces its efficiency causing amplification failure in PCR processes (Smith et al., 2002; Wu et al., 2009). Hybridization detection by biosensors showed that the device is effective enough to discriminate single nucleotide polymorphism (Dubertret et al., 2001; Maxwell et al., 2002; Kim et al., 2011). Thus, DNA markers containing $\geq 10 \%$ mismatches should perform well in biosensor-based hybridization detection. However, the performances of the probes developed should be tested in real-experiment to eliminate any doubts or ambiguities about their efficacy and effectiveness.

\section{Detection and quantitation principle of the hybrid nanobioprobe}

It has been shown that hybrid materials composed of single-stranded DNA, covalently linked to a small gold nanoparticle (2-3 nm in diameter) via sulfur-gold bond at one extremity and a fluorescent dye at the other, can assume two distinct conformations: 1) a constrained conformation (stem-loop or arch-like structure in closed state) that appears before target binding, and 2) a rod-like structure (straight conformation in open state) that appears after target binding. In the constrained structure, the fluorophore and the GNP are held in close proximity and the fluorescence is quenched by non-radiative energy transfer from dye to the metal. On the other hand, in the rod-like structure, the fluorophore is far $(>2 \mathrm{~nm})$ from the metal particle and emits fluorescence (Dubertret et al., 2001; Gerion et al., 2002, Maxwell et al., 2002). We assume that the degree of fluorescence emission depends on the degree of target binding. Thus, the maximum fluorescence should be observed when the probe is saturated with the targets and the baseline fluorescence should be obtained in the absence of any targets. Based on this assumption, a standard curve could be generated with known concentrations of probes and targets, and the concentration of the unknown could be obtained by plugging the observed fluorescence in the standard curve. The concentration of unknown sample should also be mathematically calculated using the following equation within a certain limit: $\mathrm{C}_{2}=$ $\mathrm{C}_{1} \mathrm{I}_{2} \mathrm{I}_{1}^{-1}$, where $\mathrm{C}_{2}$ is the concentration of unknown target below the saturation limit; $\mathrm{I}_{2}$ is the observed fluorescence of the unknown; $\mathrm{C}_{1}$ is the concentration of the standard target below the limit of saturation, and $\mathrm{I}_{1}$ is the fluorescence intensity of the standard. Using the formula and closely matched values of the standard in a standard curve, the calculated concentrations of the unknown are found to be fairly accurate. The operating principles of the nanoparticle probes are schematically shown in Figure 1.

\section{Specificity of the prepared nanobioprobe}

The fluorescence spectra of $10 \mathrm{nM}$ porcine nanobiosensor probes with 4-fold molar excess $(60 \mathrm{nM})$ of complementary, single-mismatch and non-complementary targets are shown in Figure 2. While the non-complementary targets produced no effect on fluorescence emission, single-mismatch targets reduced the fluorescence of the perfectly match targets by $65-70 \%$, demonstrating that the fabricated nanobiosensor probe was highly specific in discriminating against the non-complementary and single-mismatch sequences. Maxwell et al. (2002) achieved 55\% quenching with 2.5-nm gold nanoparticle probes, where gold nanoparticles were produced by sodium borohydride reduction. Dubertret et al. (2001) achieved 75\% reduction in fluorescence with molecular beacon and 1.4-nm gold nanocrystals. According to 


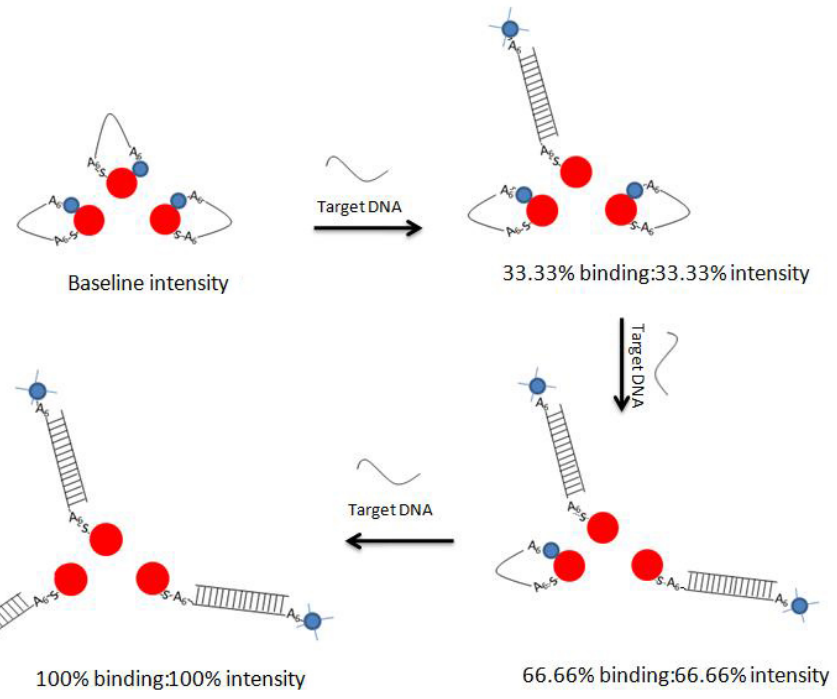

Figure 1. Schematic representations of quantification and operating principles of swine nanobiosensor probe. Three oligonucleotide probes flanked by a hexyl-A spacer at both sides and alkanethiol cap at one end and fluorophore at the other are shown to self-organize in a constrained arch-like structure, where the fluorophore is quenched by the nanoparticle in the absence of any complementary targets. Upon target binding, the closed structure is opened into a rod-like conformation separating the fluorescent dye from the nanoparticle to allow fluorescence emission. The degree of fluorescence emission is shown to be proportional to the degree of target hybridization, constituting the basis of quantitation. Gold nanoparticles and fluorophores are demonstrated by red and blue spheres. Single-strand DNA and double-strand DNA are represented by single and cross-linked double lines.

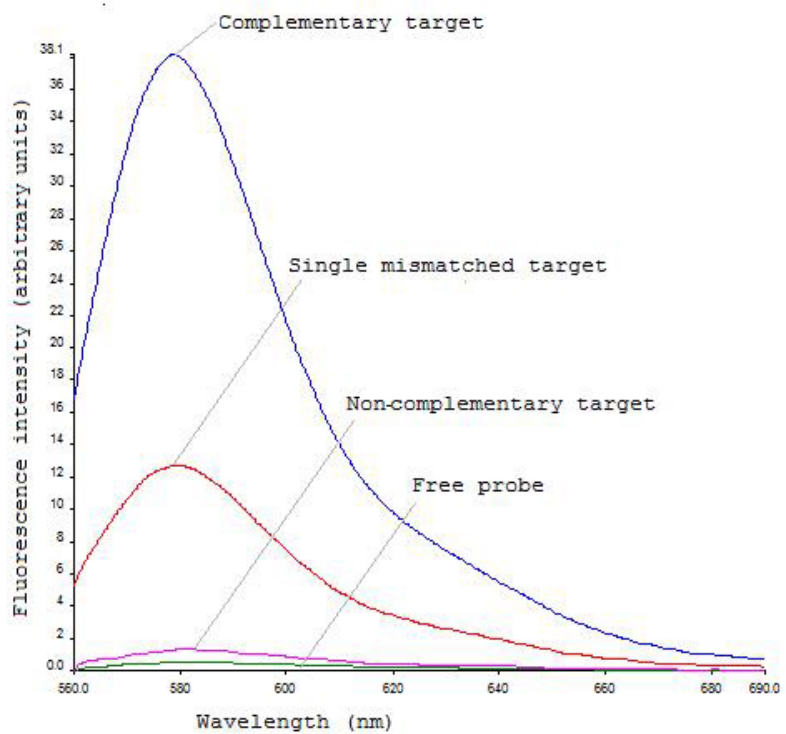

Figure 2. Detection of specific DNA sequences and single nucleotide mismatches using porcine-specific nanobiosensor probes $(10 \mathrm{nM})$. From top to bottom are 4-fold excesses (in terms of copy number) $(60 \mathrm{nM})$ : perfectly complementary (blue curve); single nucleotide mismatch (red curve); non-complementary targets (pink curve). The emission spectrum of free nanobiosensor probe $(10 \mathrm{nM})$ is shown as green curve (the bottom one). 
the latter group, low ionic strength hybridization buffer $(90 \mathrm{mM} \mathrm{KCl}, 10 \mathrm{mM}$ Tris, $\mathrm{pH}$ 8.0) differentiate perfectly matched and mismatched sequences more precisely at ambient temperature. Although, the latter group achieved higher sensitivity, the gold particles they used were too small and unstable above $50^{\circ} \mathrm{C}$. Using the relatively more stable citrate-tannate coated gold nanoparticles with a relatively large diameter (Srisombat et al., 2008) as well as low ionic strength hybridization buffer (Dubertret et al., 2001), we achieved a specificity that was higher than that of Maxwell et al. (2002) and close to that of Dubertret et al. (2001).

\section{Specificity in mixed meats}

Since commercial meat products contain mixed DNAs, binary meat admixtures were prepared by spiking $50,25,10,5,3$, and $1 \%(\mathrm{w} / \mathrm{w})$ pork into beef in a $100 \mathrm{~g}$ portion. Similar portion of $100 \%$ pork and $100 \%$ beef were used as control. All the mixtures and pure meats were extensively autoclaved for $2.5 \mathrm{~h}$ at $120^{\circ} \mathrm{C}$ at $45 \mathrm{psi}$ prior to DNA extraction as such treatment is reported to breakdown DNA, causing failure of the PCR-based meat identifications (Ali et al., 2011b,d; Yusop et al., 2011). The fluorescent spectra of AluI-digested DNAs of various mixtures including pure pork, beef, chicken, mutton, and chevon are shown in Figure 3. The sequences of all the relevant species are also shown in the inset. Detectable fluorescence was obtained only from pure pork and pork containing mixtures carrying as low as $1 \%$ pork-adulterated meat. Fluorescence profiles of meat from other species were similar or very close to the baseline fluorescence of the free probe, reflecting its strong specificity for pork. No cross-species fluorescence was observed in triplicate determinations showing an excellent reliability of the assay to determine pork in commercial meats and meat products.

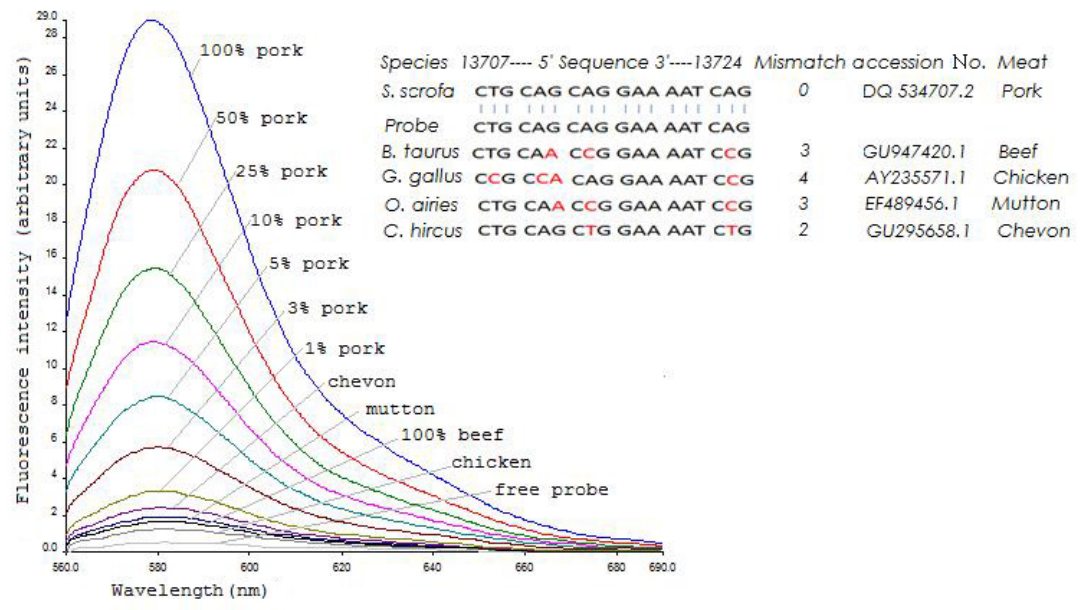

Figure 3. Pork detection in 2.5-h autoclaved pork-beef mixtures and pure meats of four common meat species under identical conditions. The corresponding emission spectra are shown by labels. The inset is the comparison of nucleotide sequences of different species with swine oligo probe. The mismatched bases are shown in red letters.

\section{Pork quantification in autoclaved pork-beef binary admixtures}

For quantification, 579-nm fluorescence intensities of AluI-digested DNAs of various 
pork-beef binary admixtures were plotted against the logarithmic values of contaminated pork $(\% \mathrm{w} / \mathrm{w})$. A sigmoidal relationship was obtained with $1-100 \%$ adulterated pork and is shown in Figure 4A. However, a fairly linear curve $\left(\mathrm{R}^{2}=0.968\right)$ was realized when the plot was constructed with $3-100 \%$ of pork in 2.5 -h autoclaved pork-beef binary admixtures. Calculation of standard error revealed higher deviations at low concentrations, reflecting inadequate probe-target collision to bring about effective hybridization. The details of the calculation are given in Table 3 .

For validation experiment, fluorescent intensities at $579 \mathrm{~nm}$ obtained from a new set of pork-beef mixtures were recorded in triplicates and the amount of pork DNA was calculated by comparing the standard set. The recovered value was plotted against the actual value and is shown in Figure 4B. A very high linearity $\left(\mathrm{R}^{2}=0.999\right)$ and very low root mean square error of calibration $(\mathrm{RMSEC}=0.011)$ were obtained, showing the high accuracy of the method in determining pork in mixed meat mixtures (Rohman et al., 2011). The details of the calculation are shown in Table 4.
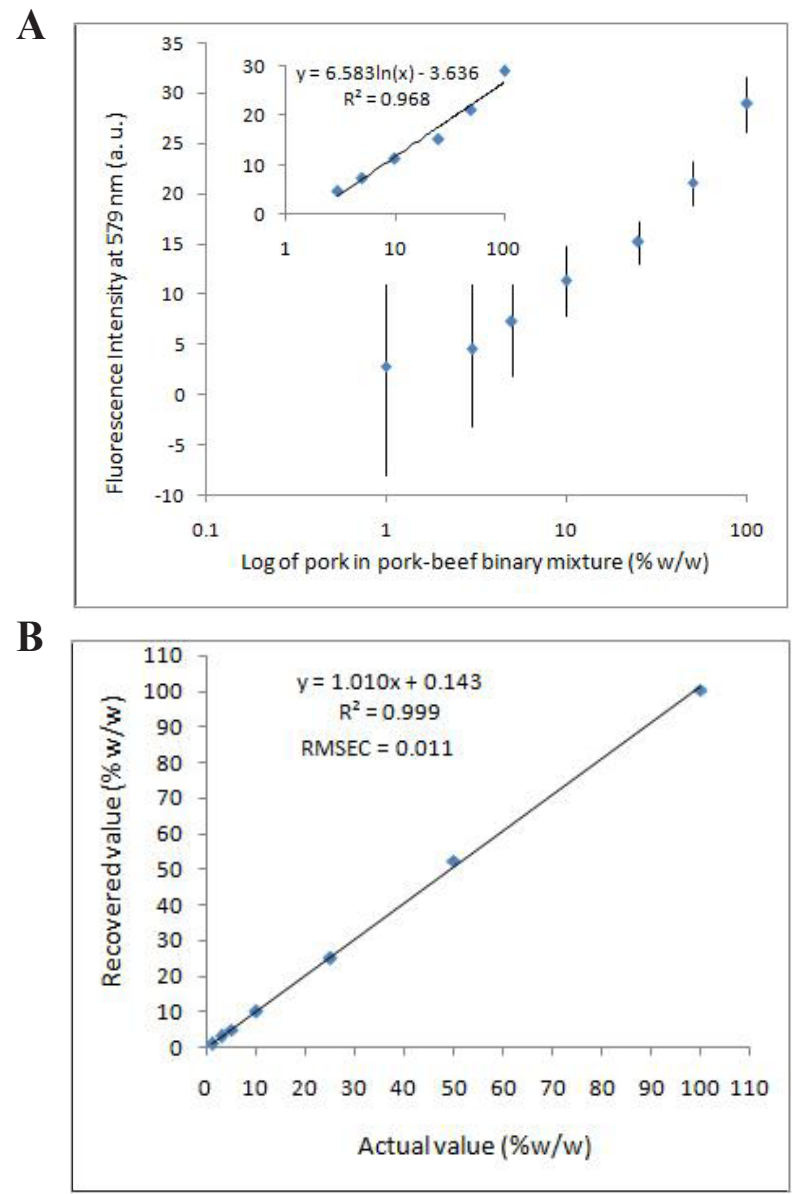

Figure 4. Pork estimation in autoclaved pork-beef binary-admixtures (A) and relationship between actual (x-axis) and recovered values (y-axis) obtained from standard and validation sets of the same mixtures (B). The standard errors (\%) are shown by the error bars and the linear part of the curve is displayed in the inset in A. 
Table 3. Data used for the generation of standard curve for quantification of pork in pork-beef binary admixtures.

\begin{tabular}{lccccccc}
\hline Pork DNA $(\mu \mathrm{g} / \mathrm{mL})$ & Fl-1 & Fl-2 & Fl-3 & \pm SD & Standard error $(\%)$ & Pork $(\%)$ & Average Fl \\
\hline 60 & 29.8 & 28.86 & 28.2 & 0.804072965 & 2.777134349 & 100 & 28.9533333 \\
30 & 21.6 & 20.98 & 20.7 & 0.460579345 & 2.183530398 & 50 & 21.0933333 \\
15 & 15.5 & 14.87 & 15.2 & 0.315119025 & 2.074516294 & 25 & 15.19 \\
6 & 11.64 & 10.88 & 11.45 & 0.39551654 & 3.492933823 & 10 & 11.3233333 \\
3 & 7.75 & 7.2 & 6.98 & 0.39661064 & 5.425590155 & 5 & 7.31 \\
1.8 & 4.9 & 4.6 & 4.2 & 0.351188458 & 7.690258214 & 3 & 4.56666667 \\
0.6 & 3.1 & 2.9 & 2.5 & 0.305505046 & 10.78253105 & 1 & 2.83333333 \\
\hline
\end{tabular}

$\mathrm{SD}=$ standard deviation.

Table 4. Quantification of pork in the validation set of autoclaved pork-beef binary admixtures.

\begin{tabular}{lllcccccc}
\hline FI-1 & Fl-2 & Fl-3 & Actual pork $(\mu \mathrm{g} / \mathrm{mL})$ & Recovered & Average Fl & Actual $(\%)$ & Recovered $(\%)$ & Standard error $(\%)$ \\
\hline 28.8 & 29.56 & 28.8 & 60 & 60.214162 & 29.053333 & 100 & 100.35694 & 0.3569372 \\
21.07 & 21.7 & 20.52 & 30 & 31.503235 & 21.096667 & 50 & 52.505392 & 5.0107848 \\
15.35 & 14.98 & 15.4 & 15 & 15.052666 & 15.243333 & 25 & 25.087777 & 0.3511082 \\
11.46 & 10.77 & 11.52 & 6 & 5.9628975 & 11.25 & 10 & 9.9381625 & -0.6183746 \\
7.65 & 7.39 & 7.08 & 3 & 3.0259918 & 7.3733333 & 5 & 5.0433197 & 0.8663931 \\
4.78 & 4.68 & 4.35 & 1.8 & 1.8131291 & 4.6033333 & 3 & 3.0218818 & 0.7293946 \\
3.15 & 3.07 & 2.48 & 0.6 & 0.614841 & 2.9 & 1 & 1.024735 & 2.4734982 \\
\hline
\end{tabular}

Quantification was performed using the average fluorescence of Table 3 as reference.

\section{CONCLUSION}

Five swine mt-genome-specific DNA markers suitable for biosensor application in terms of specificity and lengths were developed. Theoretical studies showed that all the probes contained at least $10 \%$ mismatches with closely related species. DNA markers bearing this extent of polymorphism should be better at discriminating species in biosensing devices, which are highly sensitive in discriminating even single-base mismatches (single nucleotide polymorphism). To support the theoretical studies, a swine-specific hybrid nanobioprobe was constructed by the covalent integration of an 18-nucleotide swine probe to a 3-nm gold nanoparticle. The probe successfully detected and quantified the presence of $1 \%$ pork DNA in extensively autoclaved pork-beef binary admixtures. No cross-species detection was recorded in a model experiment with four most common meat species, suggesting that the hybrid nanobioprobe could be used for the analysis of pork adulteration in halal and kosher meats and meat products.

\section{ACKNOWLEDGMENTS}

Y.B. Che Man and U. Hashim were recipients of RUGS (\#9031) and MOSTI grants (\#05-01-35-SF-1030), respectively. M.E. Ali is the recipient of a Graduate Assistantship from Universiti Malaysia, Perlis.

\section{REFERENCES}

Aboud MJ, Gassmann M and McCord BR (2010). The development of mini pentameric STR loci for rapid analysis of forensic DNA samples on a microfluidic system. Electrophoresis 31: 2672-2679. 
Ali ME, Hashim U, Dhahi TS, Mustafa S, et al. (2011a). Analysis of pork adulteration in commercial burgers targeting porcine-specific mitochondrial cytochrome B gene by TaqMan probe real-time polymerase chain reaction food. Food Anal. Methods DOI: 10.1007/s12161-011-9311-4.

Ali ME, Hashim U, Mustafa S and Che Man YB (2011b). Swine-specific PCR-RFLP assay targeting mitochondrial cytochrome B gene for semiquantitative detection of pork in commercial meat products. Food Anal. Methods 5: 613-623.

Ali ME, Hashim U, Mustafa S, Che Man YB, et al. (2011c). Nanoparticle sensor for label free detection of swine DNA in mixed biological samples. Nanotechnology DOI: 10.1088/0957-4484/22/19/195503.

Ali ME, Hashim U, Mustafa S, Che Man YB, et al. (2011d). Nanobiosensor for detection and quantification of DNA sequences in degraded mixed meats. J. Nanomater. DOI: 10.1155/2011/781098.

Ali ME, Hashim U, Mustafa S, Man YBC, et al. (2011e). Nanobiosensor for the detection and quantification of specific DNA sequences in degraded biological samples. IFMBE Proceed. 35: 384-387.

Ali ME, Hashim U, Mustafa S, Che Man YB, et al. (2012). Gold nanoparticle sensor for the visual detection of pork adulteration in meatball formulation. J. Nanomater. DOI: 10.1155/2012/103607.

Bielikova M, Pangallo D and Turna J (2010). Polymerase chain reaction - restriction fragment length polymorphism (PCR-RFLP) as a molecular discrimination tool for raw and heat-treated game and domestic animal meats. J. Food Nutr. Res. 49: 134-139.

Butler JM (2005). Forensic DNA Typing-Biology, Technology and Genetics of STR markers. 2nd edn. Elsevier, New York. Butler JM (2006). MiniSTRs: Past, Present, and Future. October 2006. Forensic News, Applied Biosystems, Foster City.

Che Man YB, Mustafa S, Khairil Mokhtar NF, Nordin R, et al. (2010). Porcine-specific polymerase chain reaction assay based on mitochondrial D-loop gene for the identification of pork in raw meat. Int. J. Food Proper. 15: 134-144.

Doosti A, Ghasemi Dehkordi P and Rahimi E (2011). Molecular assay to fraud identification of meat products. J. Food Sci. Technol. DOI: 10.1007/s13197-011-0456-31-5.

Dubertret B, Calame M and Libchaber AJ (2001). Single-mismatch detection using gold-quenched fluorescent oligonucleotides. Nat. Biotechnol. 19: 365-370.

Gerion D, Parak WJ, Williams SC, Zanchet D, et al. (2002). Sorting fluorescent nanocrystals with DNA. J. Am. Chem. Soc. 124: 7070-7074.

Hill CR, Coble MD and Butler JM (2006). Development of 27 New MiniSTR Loci for Improved Analysis of Degraded DNA Samples. American Academy of Forensic Sciences, February 24, 2006, Poster B2105, Seattle.

Hird H, Chisholm J, Sanchez A, Hernandez M, et al. (2006). Effect of heat and pressure processing on DNA fragmentation and implications for the detection of meat using a real-time polymerase chain reaction. Food Addit. Contam. 23: $645-650$.

Kim C-H, Jung C, Lee K-B, Park HG, et al. (2011). Label-free DNA detection with a nanogap embedded complementary metal oxide semiconductor. Nanotechnology DOI: 10.1088/0957-4484/22/13/135502.

Luo A, Zhang A, Ho S, Xu W, et al. (2011). Potential efficacy of mitochondrial genes for animal DNA barcoding: a case study using eutherian mammals. BMC Genomics 12: 84.

Maxwell DJ, Taylor JR and Nie S (2002). Self-assembled nanoparticle probes for recognition and detection of biomolecules. J Am. Chem. Soc. 124: 9606-9612.

Mirkin CA, Letsinger RL, Mucic RC and Storhoff JJ (1996). A DNA-based method for rationally assembling nanoparticles into macroscopic materials. Nature 382: 607-609.

Mozayani A and Noziglia C (2010). The Forensic Laboratory Handbook - Procedures and Practice. Humana Press, Houston, 30-41.

Rohman A, Sismindari, Erwanto Y and Che Man YB (2011). Analysis of pork adulteration in beef meatball using Fourier transform infrared (FTIR) spectroscopy. Meat Sci. 88: 91-95.

Smith S, Vigilant L and Morin PA (2002). The effects of sequence length and oligonucleotide mismatches on 5' exonuclease assay efficiency. Nucleic Acids Res. 30: e111.

Srisombat LO, Park JS, Zhang S and Lee TR (2008). Preparation, characterization, and chemical stability of gold nanoparticles coated with mono-, bis-, and tris-chelating alkanethiols. Langmuir 24: 7750-7754.

Wu JH, Hong PY and Liu WT (2009). Quantitative effects of position and type of single mismatch on single base primer extension. J. Microbiol. Methods 77: 267-275.

Yusop M, Mustafa S, Che Man Y, Omar A, et al. (2011). Detection of raw pork targeting porcine-specific mitochondrial cytochrome B gene by molecular beacon probe real-time polymerase chain reaction. Food Anal. Methods DOI: 10. 1007/s12161-011-9260-y. 